See Article page 285 .

\section{Commentary: Pleurography: We can, but should we?}

\author{
Cecilia Benz, MD, and Ikenna Okereke, MD
}

Various methodologies for detecting air leaks have been described in the literature, including intraoperative and radiologic strategies. In the study by Watanabe and colleagues, ${ }^{1}$ the authors revisit pleurography as a safe and effective method for localizing prolonged air leaks. Existing data is clear that patients with prolonged air leaks have an longer hospital length of stay as well as higher overall treatment costs. ${ }^{2}$ This approach offers a protocol for prompt radiologic diagnosis to optimize operative intervention for pleural fistulae.

Inclusion criteria for this study included patients with recurrent primary and secondary spontaneous pneumothorax. To adequately investigate the pleural cavity, optimal tube thoracostomy position and function are essential. Patients with bullous pulmonary disease may require multiple tubes for satisfactory reexpansion and thus need a modified protocol for sequential instillation of contrast into each tube. This modification could also be applied to patients with postoperative air leak following lobectomy or segmentectomy, a common and potentially bothersome complication. ${ }^{3}$

Although the contrast used in this study resulted in few complications, the study is limited in its evaluation of the entire lung parenchyma. The authors acknowledge that visualization of air leaks is poorest at the lung apex and centrally near the hilum. Watanabe and colleagues ${ }^{4}$ described using saline-filled computed tomography thoracography for preoperative localization of air leaks. By combining

From the Division of Cardiothoracic Surgery, University of Texas Medical Branch, Galveston, Tex.

Disclosures: The authors reported no conflicts of interest.

The Journal policy requires editors and reviewers to disclose conflicts of interest and to decline handling or reviewing manuscripts for which they may have a conflict of interest. The editors and reviewers of this article have no conflicts of interest.

Received for publication March 22, 2021; revisions received March 22, 2021; accepted for publication March 25, 2021; available ahead of print March 30, 2021.

Address for reprints: Ikenna Okereke, MD, Division of Cardiothoracic Surgery, University of Texas Medical Branch, 301 University Blvd, Galveston, TX 77555 (E-mail: ikokerek@utmb.edu).

JTCVS Techniques 2021;7:292-3

2666-2507

Copyright (C) 2021 The Author(s). Published by Elsevier Inc. on behalf of The American Association for Thoracic Surgery. This is an open access article under the CC BY-NC-ND license (http://creativecommons.org/licenses/by-nc-nd/4.0/).

https://doi.org/10.1016/j.xjtc.2021.03.029
Check for updates

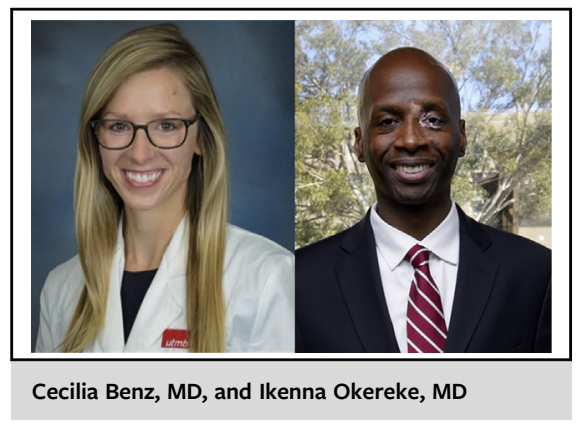

CENTRAL MESSAGE

Prolonged air leak can be a

bothersome complication. The

use of pleurography as part of a

multimodal approach to local-

izing air leaks can improve out-

comes and decrease the costs of

care.

these 2 approaches through utilization of computed tomography thoracography with nonionic iodine-based contrast, routine complete evaluation of the pleural space might be achieved.

Fluoroscopy is an inherently low-cost study, but implementation of the described pleurography protocol would require specialized training for radiology technicians, as this procedure is used infrequently worldwide. This training would include instructions on patient positioning to visualize all areas of the pleural cavity and guidelines for managing the drainage catheter during the procedure.

With the addition of pleurography, the authors hope to minimize the length of operative time and maximize surgeon efficiency by optimizing location and size of incisions. Although their data do not indicate a statistically significant decrease in operative time for patients who underwent pleurography, visualization of the pleural space does provide invaluable information for the surgeon. It is our opinion that pleurography should not be considered in isolation; instead, other points of evidence, such as previous imaging studies, clinical data, and patient factors, should be used together before taking a patient to the operating room. Pleurography should be considered as part of a multimodal strategy for minimizing hospital-associated costs and improving outcomes for patients undergoing thoracic surgery. 


\section{References}

1. Watanabe K, Imamura N, Yusa J, Koezuka S, Yamanaka S. Pleurography (thoracography) for pleural fistulas: a case series. J Thorac Cardiovasc Surg Tech. 2021;7:285-91.

2. Yoo A, Ghosh S, Danker W, Kassis E, Kalsekar I. Burden of air leak complications in thoracic surgery estimated using a national hospital billing database. Clinicoecon Outcomes Res. 2017;9:373-83.
3. Brunelli A, Cassivi S, Halgren L. Risk factors for prolonged air leak after pulmonary resection. Thorac Surg Clin. 2010;20:359-64.

4. Watanabe T, Noda M, Okazaki T, Tsukidate H, Sato K, Notsuda H, et al. Preoperative saline-filled computed tomography thoracography for awake video-assisted thoracic surgery: report of three cases. Surg Today. 2015;45: 1579-82. 